


ISSN 2524-9347

Grupo de Estudios sobre Asia y América Latina Instituto de Estudios de América Latina y el Caribe Universidad de Buenos Aires

EU

DE BA 
Un clima para el cambio. Oportunidades y desafíos para la articulación interregional ASEAN-MERCOSUR en materia de cambio climático

DiEgo TOLEDO

\section{UN CLIMA PARA EL CAMBIO. OPORTUNIDADES Y DESAFÍOS PARA LA ARTICULACIÓN INTERREGIONAL ASEAN- MERCOSUR EN MATERIA DE CAMBIO CLIMÁTICO}

\section{Diego Toledo}

Universidad Católica de Córdoba

toledo_bugarini@hotmail.com

\section{Introducción}

El cambio climático se ha convertido en uno de los principales temas de la agenda política global. En la medida en que los plazos para afrontar el problema son cada vez más reducidos y sus consecuencias se hacen más notorias, se vuelven más relevantes los estudios sobre el tema. Para el caso de los países del Sur Global, los incrementos de los impactos conllevan una profundización de los problemas ya existentes en nuestras sociedades como son las desigualdades multidimensionales, la forma de vinculación con el ambiente y el engranaje productivo-comercial.

El presente trabajo pretende abordar las relaciones ASEANMERCOSUR desde una perspectiva interregional y un nivel macro de análisis, enfatizando en la temática del cambio climático y exponiendo un marco general de posibilidades que se abren para la cooperación sur-sur entre ambos bloques o entre sus Estados parte. Esto debido a que se entiende que, hasta el momento, las iniciativas interregionales entre ambos bloques funcionan como un complemento al bilateralismo entre Estados de América del Sur y del Sudeste Asiático (SEA en adelante) (Rubiolo, 2016).

La elección del tema responde no solo a la relevancia política de la agenda del cambio climático y su integración con la agenda social y gubernamental de los países mencionados, sino que además espera resultar innovadora en tanto se constata que la mayoría de los estudios que abordan las relaciones SEA-América del Sur se dirigen al análisis de las transformaciones, desafíos y potencialidades del flujo e intercambio comercial entre países de ambas regiones (Ramoneda, 2013).

El diseño metodológico es de tipo cualitativo. Se emplean técnicas de recolección de datos provenientes de fuentes primarias (documentos oficiales de ASEAN, MERCOSUR) y secundarias (artículos publicados en revistas científicas, policy briefs de organismos especializados en la temática, etc.). El análisis documental, tanto estadístico como cualitativo, es la principal técnica de análisis de información. No existe un recorte temporal explícito dado que entiende la construcción institucional y de agenda de ambos bloques como un 
Un clima para el cambio. Oportunidades y desafíos para la articulación interregional ASEAN-MERCOSUR en materia de cambio climático

DiEgo TOLEDO

proceso abierto. Sin embargo, el lector encontrará que los principales desarrollos parten desde inicios de este siglo hasta el presente (2000-2019).

El texto, entonces, se estructura en torno a cuatro apartados. El primero, plantea algunas notas sobre el contexto global en el cual se despliegan las relaciones interregionales. El segundo, expone algunos apuntes en torno a la gobernanza global del cambio climático y explora el posicionamiento y la situación de los países ASEAN-MERCOSUR en relación con la temática. Luego, se presentan las trayectorias que los países de ASEAN y del MERCOSUR han seguido para responder a las problemáticas del cambio climático, se analizan sus dificultades y se explora la posibilidad de articulaciones, transferencias y formatos de cooperación sursur entre ambos bloques. Finalmente, se presentan algunas conclusiones a modo de síntesis.

\section{Declive de Occidente, ¿̇ascenso del resto ${ }^{1}$ La relevancia de la articulación ASEAN-MERCOSUR en el actual escenario global}

El escenario internacional actual está atravesando un momento crítico, de transformación. El mundo multipolar en el que vivimos está surcado por una globalización en crisis y por una disputa por el liderazgo mundial. Hasta el momento, la crisis de la globalización conlleva movimientos paradójicos. Mientras siguen intentándose algunos avances en mega acuerdos o iniciativas de "regionalismo del siglo XXI" (Baldwin, 2011; Zelicovich, 2016), se percibe el "retorno" al proteccionismo y un inquietante ascenso de nacionalistas de derecha en diversas partes del globo (Sanahuja, 2019). Estos cambios vienen acompañados de una revitalización de los acuerdos bilaterales de libre comercio y de virajes en las formas del multilateralismo, que transita desde "lo abierto e incluyente" -influido por el fracaso de las negociaciones en las rondas de la Organización Mundial del Comercio (OMC) - hacia los "Foros G" (Sanahuja 2019) o alternativas de multilateralismo corporativo (Tussie, 2019).

En paralelo, se experimenta el desarrollo y convergencia de nuevos actores emergentes -como los denominados BRICS (Brasil, Rusia, India, China y Suráfrica) - y el desenvolvimiento de diversos polos de poder frente a las hegemonías "tradicionales" o históricas de Estados Unidos y Europa.

\footnotetext{
${ }^{1}$ El título del apartado pone en clave interrogativa una afirmación del analista Barry Buzan. Considero que el tono cuestionador resulta una forma más propicia para el análisis que, además, evita caer en interpretaciones proclive al "choque de civilizaciones" o la "Trampa de Tucídides", que nos lleva a creer que las respuestas o disuasión armada son la solución a un contexto de crisis o transición hegemónica (bloque histórico hegemónico).
} 
Un clima para el cambio. Oportunidades y desafíos para la articulación interregional ASEAN-MERCOSUR en materia de cambio climático

Diego Toledo

Particularmente, el actual enfrentamiento entre China y Estados Unidos -que excede lo comercial ${ }^{2}$ - juega un rol fundamental en el reordenamiento geoeconómico y político a nivel mundial.

Para el caso latinoamericano, la presencia de China era algo prácticamente insignificante hasta el inicio del nuevo milenio. Con el ingreso de China a la OMC en 2001, el cambio comenzó a gestarse (Ramón Berjano, 2019, p. 37). Desde entonces, China se ha vuelto el primer o segundo socio comercial de los países latinoamericanos y se ha convertido en la principal fuente de financiamiento de proyectos de desarrollo regional, por encima de los organismos tradicionales como el BID o el BM (Tussie, 2019, p. 110). Hasta tal punto llega la interconexión que, en 2017, durante el Foro de la Iniciativa de la Ruta de la Seda en Beijing el presidente Xi Jinping llegó a referirse a la región como "la extensión natural" de la Iniciativa (Ramón Berjano, 2019, p. 43).

El aumento de esta interdependencia latinoamericana hacia China (Piccone, 2016), y el hecho de que los países latinoamericanos definitivamente no pueden dejar de colocar sus materias primas en el mercado chino (Tussie, 2019, p. 113) genera, como contraparte, una presión más fuerte hacia América Latina por parte de los Estados Unidos, que siente amenazada no solo su economía sino también la hegemonía en lo que históricamente ha considerado su "patio trasero". Por ello, apoyado en la doctrina del "American First" -que no es más que una reactualización histórica de la idea del Destino Manifiesto y de un sentido auto adjudicado de excepcionalidad-, Estados Unidos (con Trump a la cabeza) está reforzando su "ofensiva" hacia lo que hoy explícitamente nombra como "países de mierda". Es importante anotar aquí algunos antecedentes que juegan un rol en este aspecto. El rechazo al ALCA (2005) y el desarrollo de iniciativas regionales como ALBA-TCP y la UNASUR (particularmente su Consejo de Defensa) supusieron trabas concretas al ejercicio de influencia estadounidense en la región. Sin embargo, con el actual declive del proyecto post-liberal de integración y el desplazamiento de la gran mayoría de gobiernos progresistas que le dieron sustento durante la década del 2000, Estados Unidos encuentra una nueva correlación de fuerzas en la que puede recuperar su influencia.

\footnotetext{
2 De acuerdo con Tussie (2019, p. 108-109) "China hoy representa el centro de una histórica transformación del sistema contemporáneo”. Además del exponencial crecimiento de su PIB, se convirtió en uno de los principales consumidores de energía eléctrica del planeta, sacó a 740 millones de personas de la pobreza, y se está posicionando como un gran acreedor mundial con la acumulación de U\$S3 trillones de reservas internacionales, diplomacia financiera que utiliza para generar simpatías y para reducir la eficacia del "castigo financiero" de Estados Unidos como medida de presión internacional.
} 
Un clima para el cambio. Oportunidades y desafíos para la articulación interregional ASEAN-MERCOSUR en materia de cambio climático

DIEGO TOLEDO

$\mathrm{Al}$ mismo tiempo, y con ayuda de think tanks conservadores, Estados Unidos intenta expandir la interpretación de la presencia china en la región como una nueva forma de colonialismo (Tussie, 2019). Pero, la permanencia de un modelo extractivista no puede adjudicarse únicamente a la profundización de las relaciones comerciales con China. La acusación de colonialismo no encuentra demasiado sustento porque China no muestra interés en "exportar" su propio modelo político, económico o social. De hecho, para la diplomacia china una asociación estratégica se basa en la complementariedad y la cooperación, y no se dirige contra un tercer Estado y tiene eje en la economía (Tussie, 2019, p. 111). Esa es su principal diferencia con Estados Unidos.

Asimismo, en el Sudeste Asiático se perciben tensiones similares en torno al enfrentamiento China-Estados Unidos. La vecindad con China y su rol hegemónico en la región implica para ASEAN una alianza ineludible que convive con el surgimiento de contrapesos políticos dentro y fuera de la región, principalmente de India, Rusia, y Estados Unidos (Buzan, 2012). La presencia de Estados Unidos en el SEA sigue replicando la lógica de la Guerra Fría y, en tanto el Acuerdo Trans Atlántico de Comercio e Inversión (TTIP) que marcó el "pivote a Asia" en 2008 hoy se encuentra en pausa, el mantenimiento de las "áreas de influencia" corre por la vía de las relaciones bilaterales.

Cabe mencionar que, si bien la integración latinoamericana eventualmente "responde" a los mencionados enfrentamientos hegemónicos globales con iniciativas más favorables o menos hacia Asia o hacia Estados Unidos-Europa; el estado heterogéneo, complejo y lleno de altibajos de la integración regional nos habla de un panorama que excede la "fractura Atlántico-Pacifico" (Sanahuja, 2016). En este sentido, se observa la coexistencia de proyectos como CAN (año), MERCOSUR (1991), ALBATCP (2004), UNASUR (2008), Alianza del Pacífico y CELAC (2011), PROSUR (2019).

Por su parte, el SEA transita transformaciones profundas relacionadas con la profundización de ASEAN como bloque, el despliegue de una nueva etapa de desarrollo económico en China, y los avances en las mega negociaciones -como el Trans-Pacific Partnership (TPP) suscrito, pero no vigente, el mencionado TTIP, y el Regional Comprehensive Economic Partnership (RCEP) impulsado por China que vincula toda la región de Asia Pacífico (ASEAN +6) (Bartesaghi, 2015).

En este contexto, las relaciones entre ambos bloques pueden contribuir a fortalecer los respectivos regionalismos "por medio del interregionalismo" (Hanggi, 2003), generando diálogos, debates e informaciones "de nivel medio" que luego pueden nutrir los procesos multilaterales mundiales, o bien producir agendas compartidas y combinadas 
Un clima para el cambio. Oportunidades y desafíos para la articulación interregional ASEAN-MERCOSUR en materia de cambio climático

DiEgo TOLEDO

con mayor o nivel de consenso que pueden también expresarse en instancias y negociaciones globales (Rubiolo, 2016).

Pese a que existen diferencias significativas en torno al modo de cooperar ${ }^{3}$, las potencialidades tampoco pueden negarse. En este sentido, aunque -por el momento- se trate de desenvolver un "interregionalismo flexible", en el que ninguna de las dos partes actúa con la otra "como si fuera un todo unificado", el desarrollo de la cooperación sur-sur puede resultar beneficiosa para ambas partes frente a posibles "estrangulamientos" de los principales polos del poder mundial como China (Lo Brutto, 2015), Estados Unidos, Rusia, etc. De acuerdo con Rubiolo (2016), el interregionalismo flexible se diferencia de los casos de interregionalismo puro o bilateral (por ejemplo. UE-ASEAN) por acoger relaciones de distinto nivel de institucionalización regional, haciendo parte de relaciones bilaterales (EstadoEstado), región-Estado, o la formación de grupos interregionales, como el caso de FOCALAE. Por ello, se considera que este marco analítico resulta más útil que el interregionalismo puro para entender las relaciones Sur-Sur o no triádicas (aquellas que no refieren a América del Norte, Europa o Asia del Este).

En medio de un enfrentamiento por la hegemonía a nivel mundial, la asociación de este particular interregionalismo puede no solo poner un límite o contrapeso a dichas relaciones hegemónicas, sino además promover o facilitar la "legalización" de las relaciones internacionales y los compromisos estatales con las normas globales.

\section{El posicionamiento de ASEAN-MERCOSUR en el contexto de la gobernanza global del cambio climático. Situaciones y respuestas}

El cambio climático es, políticamente, un tema difícil por tres razones básicas (Keohane y Victor, 2010). En primer lugar, al ser un problema global no puede ser solucionado de manera unilateral o fragmentada, requiere necesariamente coordinación y acción colectiva. En segundo lugar, es un problema intergeneracional que implica un esfuerzo doble para los líderes interesados en impulsar una acción efectiva: deben persuadir a sus seguidores de que su acción marca una diferencia y de que los costos de hoy impactarán en el futuro. Por último, cambiar las prácticas de tantas personas, organizaciones, gobiernos y empresas supone para los Estados un interés y

\footnotetext{
3 Por ejemplo, Mercosur no contempla la firma de acuerdo de libre comercio individuales por parte de sus miembros mientras ASEAN sí. Además, mientras en el SEA se tiende a un regionalismo amplio, abierto y flexible, en América Latina subsiste una mayor fragmentación de mercados regionales por la competencia entre miembros del bloque (Rubiolo, 2016).
} 
Un clima para el cambio. Oportunidades y desafíos para la articulación interregional ASEAN-MERCOSUR en materia de cambio climático

DiEgo TOLEDO

una capacidad de ejercer influencia y otorgar incentivos para realizar esas transformaciones.

De acuerdo con Palacio (2013, p. 51), el cambio climático no debe ser confundido con la variabilidad climática, que refiere simplemente fenómenos naturales de variación climática, tales como El Niño, que se producen independientemente de la acción humana. Sin embargo, esto no quiere decir que estos fenómenos no estén afectando la vida en general un modo más grave a causa del cambio climático.

La Convención Marco de las Naciones Unidas sobre el Cambio Climático (CMNUCC), en su Artículo 1, define 'cambio climático' como 'un cambio de clima atribuido directa o indirectamente a la actividad bumana que altera la composición de la atmósfera mundial y que se suma a la variabilidad natural del clima observada durante períodos de tiempo comparables' (el subrayado es mío). El IPCC (2001), por su parte, define al cambio climático de una manera más amplia. Lo entiende como:

una importante variación estadística en el estado medio del clima o en su variabilidad, que persiste durante un período prolongado (normalmente decenios o incluso más). El cambio climático se puede deber a procesos naturales internos o a cambios del forzamiento externo, o bien a cambios persistentes antropogénicos en la composición de la atmósfera o en el uso de las tierras. (p. 175)

En tanto fenómeno de afectación global, la problemática del cambio climático viene siendo objeto de variados intentos de regulación y acción en la esfera internacional. Por ello, el cambio climático tiene una estructura de gobernanza global que le es propia. En términos de Ruland y Storz (2008) un sistema de gobernanza global es "una articulación de instituciones, por lo general pero no exclusivamente gubernamentales, que regulan el comportamiento de los Estados y otros actores en diferentes áreas de la política internacional" (p. 3). No obstante, dada la complejidad que le aporta su carácter multicausal, su expresión multidimensional y la variabilidad de los compromisos asumidos por los Estados, el estado de tal gobernanza es difuso y variado.

Difuso porque se percibe, en realidad, la coexistencia de un régimen internacional formal -que encabeza la CMNUCC- y de una serie de iniciativas de actores privados, ONG globales y actores estatales subnacionales, entre otros. No hay una arquitectura global completamente integrada que cohesione todo el conjunto de iniciativas y regulaciones. En otras palabras, coexiste un régimen internacional de cambio climático con iniciativas transnacionales de gobernanza climática (Hickmann, 2017). 
Un clima para el cambio. Oportunidades y desafíos para la articulación interregional ASEAN-MERCOSUR en materia de cambio climático

DiEgo TOLEDO

$\mathrm{Y}$ variado porque, dentro del régimen internacional de cambio climático, lo que existe es una amplia gama de regímenes regulativos específicos para las distintas problemáticas (como el uso de la tierra y forestación, o las emisiones de gases de efecto invernadero), que a su vez pueden reforzarse mutuamente o bien entrar en contradicción o conflicto. De acuerdo con Keohane y Victor (2010), pese a que estos arreglos formales en la materia necesitan forjar un mayor estándar de coherencia, efectividad, determinación, sustentabilidad, accountability y "calidad epistémica"; su carácter flexible, en alcance y contenido, los vuelve útiles y políticamente realistas dada la incertidumbre y los flujos políticos y económicos que influyen sobre la acción climática en sus distintos niveles.

Comprender este "complejo de régimen" (Keohane y Victor, 2010) resulta fundamental en un contexto de transformación de la política internacional del cambio climático (del Pilar Bueno, 2012), en el que la emergencia de un enfoque bottom-up se traduce en la formulación compromisos voluntarios de las partes o, como suelen llamarse, "Contribuciones Previstas y Nacionalmente Determinadas" (iNDCs, por sus siglas en inglés) (Kiessling, 2019). Para los países en desarrollo de ambas regiones (SEA y MERCOSUR), el desafío es singular ya que implica afrontar políticas de mitigación y de adaptación a la vez, con un presupuesto por lo general limitado; lo que muchas veces genera un trade-off entre las mismas.

Estudiar el condicionamiento mutuo entre política climática y política internacional del cambio climático en las potencias o países emergentes es importante porque, como afirma Kiessling (2019):

(...) son Estados que son objeto de flujos de socialización de normas internacionales que no necesariamente son generadas domésticamente, pero que, en su internalización, a través de la localización, producen procesos de contestación que transforman el significado, la interpretación y el sentido de la norma; lo que, a través de la capacidad de agencia de estos actores, termina impactando nuevamente sobre la gobernanza global del cambio climático. (p. 80)

\footnotetext{
${ }_{4}^{4}$ Aunque existe un acuerdo generalizado con los lineamientos generales del IPCC, es bueno mencionar que algunas visiones críticas apuntan que en los estudios del IPCC hay un "eslabón ausente" en la economía del carbono. No se evalúa la capacidad de los países para capturar y almacenar carbono en sus tierras. Esto generaría un sesgo distorsivo en las políticas, que solo tendrían en cuenta la medida de las emisiones de gases. Los números se ordenan de manera radicalmente distinta cuando esas emisiones son expresadas por hectárea de tierra y no por habitante. Por ejemplo, mientras los cuatro países del Mercosur registran menos del $3 \%$ de las emisiones globales de carbono; por ser grandes secuestradores de carbono, algunos indicadores le asignan una emisión per cápita mayor que las de China e India, dos de los mayores emisores mundiales (Viglizzo y Ricard, s/f; Viglizzo, 2018).
} 
Un clima para el cambio. Oportunidades y desafíos para la articulación interregional ASEAN-MERCOSUR en materia de cambio climático

DiEgo TOLEDO

Todos los Estado miembros de ASEAN y del MERCOSUR han ratificado el Protocolo de Kioto y han firmado el Acuerdo de París. Sin embargo (con la clara y notoria excepción de Brasil o de los vecinos del SEA, China e India), ninguno parece prefigurarse como generador de normas internacionales. En su lugar, todos se posicionan como tomadores de normas, se limitan a aceptar o contestar las normas que otros Estados generan. Por ello, la cooperación entre ambas partes resulta necesaria.

Fuera de la casuística y de la heterogeneidad y especificidad con la que el cambio climático se manifiesta en cada región, los países de ambas regiones enfrentan desafíos similares en relación a los impactos que el cambio climático produce en ambas regiones: incendios forestales (Indonesia, Malasia y Singapur en 2015; la Amazonía brasilera y la Chiquitanía boliviana en 2019); inundaciones (norte de Tailandia en 2017, Argentina y Uruguay en 2017), riesgo de perder poblaciones y entramados productivos en ciudades costeras por el aumento del nivel del mar-agravados por las migraciones del campo a la ciudad-, mayor dependencia de importación de bienes básicos (para el caso del SEA), y mayores riesgos de pérdidas y crisis económicas dada la dependencia a las exportaciones de dichas materias primas en América del Sur.

Frente a esto, ambas regiones se están haciendo preguntas similares en torno a problemáticas de seguridad alimentaria, la necesidad de una transición energética (de un modelo basado en lo fósil a un modelo de energía renovable) y de modelos de desarrollo más sustentables, que dejen de estar surcados por factores como las deforestaciones y el avance de la frontera agrícola, las prácticas extractivistas o la deficitaria regulación de empresas multinacionales, entre otras.

\section{Trayectorias regionales de ASEAN y MERCOSUR en materia de cambio climático. Límites, desafíos y posibilidades para la cooperación}

ASEAN es el segundo proyecto de integración regional más antiguo después de la Unión Europea (Ramoneda, 2013; Rubiolo, 2011). Ha cooperado en materias ambientales entre sus integrantes desde 1977, reconociendo así la importancia del tema para el desarrollo sustentable y la integración regional (Association of Southeast Asian Nations, 2019a). Desde el Foro realizado en Singapur en 2007, ASEAN ha promovido la cooperación e integración entre sus Estados parte en cambio climático y ha identificado constantemente al cambio climático como una temática prioritaria de la organización (ASEAN, 2019b).

Desde el 2015, el mismo año en que las Naciones Unidas formuló los nuevos Objetivos de Desarrollo Sustentables (ODS) para una Agenda 2030, 
Un clima para el cambio. Oportunidades y desafíos para la articulación interregional ASEAN-MERCOSUR en materia de cambio climático

DiEgo TOLEDO

ASEAN realizó su tránsito hacia la "Comunidad ASEAN" (ASEAN Community), según había sido fijado por los líderes de los países integrantes en 2003.

Bajo este nuevo formato institucional, se establecieron tres pilares fundamentales sobre los que se desarrollaría la región, creando tres Comunidades: Política (Seguridad), Económica, y Socio-Cultural (ASCC, en inglés) (Asean, 2015). Esta última, supervisa y contiene todos los programas de ASEAN relativos a la cooperación y actividades conjuntas en cambio climático.

Tailandia funciona como el coordinador de ASEAN en materia de ODS y procura su alineamiento y complementariedad con las áreas prioritarias del bloque (Letchumanan, 2010), siguiendo los lineamientos del plan "ASEAN 2025 Forging Ahead Together" (ASEAN, s/f) y de la ASCC Blueprint 2025, la cual guía la cooperación en temas ambientales de la ASEAN, proyectando el fortalecimiento de una comunidad que compromete y beneficia a sus pueblos, siendo "inclusiva, sustentable, resiliente y dinámica".

La actual estructura institucional de ASEAN para la cooperación en temas ambientales está compuesta por las Reuniones Ministeriales de Ambiente de ASEAN (AMME, en inglés), que ocurren una vez cada dos años; los Oficiales Senior de Ambiente de ASEAN (ASOEN, en inglés) y siete grupos de trabajo subsidiarios, que se reúnen una vez por año para revisar la implementación del Plan Estratégico de Ambiente de ASEAN (ASPEN, en inglés) y ASCC Blueprint 2025. (Letchumanan, 2010).

Mientras la "Sección D" del ASCC Blueprint 2025 establece específicamente medidas de acción dirigidas a mitigar los impactos del cambio climático, en tanto se persigue como objetivo general "asegurar la sustentabilidad medioambiental" (ASEAN, 2016), el ASPEN está integrado por siete áreas prioritarias, correspondientes a cada uno de los grupos de trabajo especializados subsidiarios de ASOEN. De estos, uno está dedicado exclusivamente a la problemática del cambio climático (AGWCC, en inglés), funcionando como el brazo operativo de la Iniciativa ASEAN para el Cambio Climático (ACCI, en inglés) a través de la Respuesta Conjunta de ASEAN para el Cambio Climático (ASEAN, 2012).

Este grupo de trabajo especializado, establecido en 2009, ha definido cinco prioridades como propias: (i) adaptación y resiliencia, (ii) mitigación, (iii) transferencia tecnológica, (iv) financiamiento del cambio climático, y (v) coordinación multisectorial (transversal) y alianzas globales.

ASEAN cuenta con diversas políticas transversales (en función del carácter multidimensional del cambio climático) y entre algunas de ellas podemos mencionar: el Programa de Trabajo para el Acuerdo en Manejo de Desastres y Respuestas a Emergencias de ASEAN (AADMER, en inglés) 
Un clima para el cambio. Oportunidades y desafíos para la articulación interregional ASEAN-MERCOSUR en materia de cambio climático

DiEgo TOLEDO

como parte de iniciativas de adaptación al cambio climático, El Acuerdo para controlar la Contaminación Transfronteriza de HASE (AATHP, en inglés) vigente desde 2003, la Estrategia ASEAN para el Manejo de Peatland (20062020), el Marco Multisectorial de ASEAN para el Cambio Climático en Agricultura, Forestry en pos del logro de los ODS, la seguridad alimentaria y la nutrición, el Plan de Acción ASEAN para la Cooperación Energética (APAEC) adoptado en 2009 por el período 2010-2015, la creación en 2005 del Centro ASEAN para la Biodiversidad y la Iniciativa ASEAN de Ciudades Ambientalmente Sustentables (AIESC) (ASEAN, 2018; Letchumanan, 2010).

Asimismo, ASEAN cuenta desde 2012 con un Plan de Acción de Respuestas Conjuntas al Cambio Climático, en que se detallan actividades, resultados a obtener y países líderes (del bloque) encargados de su ejecución o vigilancia (ASEAN, 2012).

Si bien los países de ASEAN formularon iNDCs bajo el Acuerdo de París, la región continúa expandiendo su capacidad de generar electricidad a base de energías fósiles, lo cual daña su reputación internacional, genera dependencia de las importaciones provenientes del Medio Oriente, y no mitiga los impactos agregados del cambio climático en la región (Øverland, 2017).

Pese a su posicionamiento favorable a las normas internacionales de cambio climático, la mayoría de los países de ASEAN no han tomado roles prominentes en este campo. Un estudio del Stockholm Environment Institute (2016), dividió a 8 de los países ASEAN en 3 categorías: los pioneros de la adaptación (Filipinas y Vietnam), que toman la adaptación como una necesidad urgente y han generado nuevos arreglos institucionales para abordarla, los campeones emergentes (Camboya, Indonesia y Myanmar), que siguen de cerca a los primeros, tomando nuevos abordajes de adaptación; y los de adaptación paulatina (Laos, Malasia y Tailandia), que por tener una menor exposición a desastres han tendido a ser complacientes ante la necesidad de adaptación, con políticas desintegradas, débiles o limitadas en su impacto.

Manteniéndose como tomadores de normas, ASEAN se enfrenta a una posible pérdida de estatus si limita únicamente a seguir lo que sus Estados miembros hacen por el cambio climático, ${ }^{5}$ más aún si estos Estados fallan en lograr sus iNDCs. Por ello, especialistas en la materia indican que ASEAN puede tener problemas de acción colectiva en materia de política climática internacional: sus Estados miembro esperan que ASEAN adopte un rol más fuerte, mientras la Secretaría de ASEAN espera que sean los Estado miembro los que lideren el proceso (Øverland, 2017).

\footnotetext{
5 Un mapeo de organismos de cambio climático para cada país de ASEAN puede verse en Salamanca, A., \& Nguyen, H. (2016).
} 
Un clima para el cambio. Oportunidades y desafíos para la articulación interregional ASEAN-MERCOSUR en materia de cambio climático

DiEgo TOLEDO

La Secretaría de ASEAN puede entonces promover un espíritu de equipo, formulando por ejemplo Contribuciones Regionalmente Determinadas (RDC) para sus Estados miembro. También puede reforzar y expandir su staff especializado en cambio climático (lo cual requiere capacitaciones y financiamiento), además de reconsiderar la manera en que conduce los negocios, enfatizando en la soberanía nacional, la no interferencia y el consenso en la toma de decisiones como principios básicos.

En el caso del MERCOSUR, parece que los objetivos ambientales se piensan de forma subordinada a una lógica comercial, ${ }^{6}$ en donde la problemática ambiental aparece relacionada con posibles distorsiones en los costos de producción (y por ende en competitividad) de los miembros de cada bloque. Es decir, existe una idea de que las medidas ambientales funcionan como una traba para el desarrollo comercial de la región, y no logra percibirse la interrelación existente entre política comercial, política ambiental/climática, y desarrollo socioeconómico (Stuhldreher, 2012).

Desde los años 90, con el avance de la globalización y el "regionalismo abierto" en la región -que implicaban una influencia creciente de las relaciones externas sobre el diseño de políticas internas- se hizo necesario explicitar una entidad regional unificada a partir de la cual la región pudiera proyectarse hacia el exterior. Esto contribuyó a que, en el ámbito medioambiental, MERCOSUR buscara la participación y posicionamiento conjuntos en negociaciones internacionales. El Preámbulo del Tratado de Asunción de 1991 ya contiene algunas notas al respecto, donde la preservación del medioambiente -supeditada a los principios de gradualidad, flexibilidad y equilibrio- aparece como un medio para ampliar los mercados mediante la integración (Fedorovsky y Niglia, 2010). Otros antecedentes fueron la Declaración de Canela (1992), que establecía el principio de complementariedad entre la protección ambiental y el desarrollo económico; y la Declaración de Taranco (1995) que, explícitamente, buscaba la consolidación de posicionamientos conjuntos hacia la arena internacional.

En 1992, se avanzó a partir de una resolución del Grupo del Mercado Común (GMC), órgano operativo del MERCOSUR, que creó la Reunión Especializada de Medio Ambiente (REMA). Esta redactó las Directrices Básicas en Materia Ambiental del MERCOSUR (1994) que, aunque sin carácter vinculante, procuraban la coordinación de criterios ambientales comunes para negociar como bloque (Fedorovsky y Niglia, 2010; Stuhldreher, 2012).

En 1995, tras la Declaración de Taranco, la REMA fue reemplazada por el Subgrupo de Trabajo 6 (SGT 6), dedicado a Medio Ambiente. El grupo

\footnotetext{
${ }^{6}$ Los debates en torno a la reciente firma del Tratado UE-Mercosur y la polémica Macron-Bolsonaro ejemplifican este punto.
} 
Un clima para el cambio. Oportunidades y desafíos para la articulación interregional ASEAN-MERCOSUR en materia de cambio climático

Diego Toledo

-que aún continua vigente- elaboró una lista de Tareas Prioritarias, entre las que se planteó la posibilidad de proyectar un Instrumento Jurídico de Medio Ambiente para el MERCOSUR, el diseño de un sistema de información ambiental (SIAM, hoy vigente) y la creación de un Sello Verde MERCOSUR (Fedorovsky y Niglia, 2010).

El Acuerdo Marco sobre Medio ambiente de 2001, adoptado por medio de una decisión del CMC, fue un hito para la región, ya que ratificó los principios de la Declaración de Río (principalmente el de responsabilidades comunes pero diferenciadas) y fijó el compromiso de los Estados a cumplimentar sus acuerdos internacionales, estableciendo así la complementariedad entre política comercial y medioambiental (Stuhldreher, 2012). Siendo el primer instrumento jurídico ambiental en dicho ámbito proyecto de integración, entró en vigor en 2004. Luego de ello, se dieron algunos otros acuerdos -como la aprobación del Protocolo Adicional en Materia de Cooperación y Asistencia ante Emergencias Ambientales-, pero fueron de menor importancia y no vinculantes (Fedorovsky y Niglia, 2010).

Sin embargo, en materia de cambio climático, las menciones son pocas. En 2009 se propusieron lineamientos para formular un proyecto de cooperación de adaptación al cambio climático en el marco de la X Reunión de ministros de Medio Ambiente; y en 2010, en la XI Reunión, se discutió sobre la formulación de una posición conjunta para la Cumbre de Cancún sobre Cambio Climático (Stuhldreher, 2012).

Vemos entonces que a diferencia de ASEAN, los países del MERCOSUR no tienen un acercamiento verdaderamente regional al fenómeno del cambio climático. Aunque se ha intentado, tampoco existen pronunciamientos ni posicionamientos conjuntos (como bloque) en las negociaciones internacionales de cambio climático (Fedorovsky y Niglia, 2010). De hecho, su participación en estas instancias suele ser desigual, donde países como Brasil tienen un rol destacado y se posicionan como creadores de normas globales para el cambio climático.

Los desafíos para construir una institucionalidad climática en Sudamérica son diversos. La transversalidad de la agenda climática es uno. La asimetría de poder de las áreas a cargo de los temas climáticos frente a otras áreas de gobierno es otro. Los déficits de implementación de las políticas climáticas (por planeamiento erróneo y un bajo nivel de law enforcement) también son destacables. Por último, también entran en juego factores políticos relativos a la voluntad política y las lógicas de poder en contextos electorales, dónde la necesidad de rédito político a corto plazo se enfrenta la temporalidad de largo plazo exigida por las (además inciertas) políticas climáticas (Manzano Franco, 2016; Ryan, 2017).

La configuración de una agenda medioambiental para el MERCOSUR constituye entonces un proceso fragmentario y no completamente coherente. 
Un clima para el cambio. Oportunidades y desafíos para la articulación interregional ASEAN-MERCOSUR en materia de cambio climático

DiEgo TOLEDO

Actualmente, el mecanismo más formal en torno a las decisiones en política climática para la región está constituido las Reuniones de Ministerios de Medio Ambiente de los Estados parte del bloque, de las cuales no hay demasiada información disponible. Si bien podemos reconocer el carácter transversal del cambio climático, la escasa evidencia documental del MERCOSUR indica que la problemática se aborda de manera secundaria o residual ante otras temáticas centrales del bloque ${ }^{7}$.

Aunque algunas tareas prioritarias del STG 6, especializado en Medio Ambiente. se relacionan al cambio climático -como las Emergencias Ambientales y el Acompañamiento de la Agenda Ambiental Internacional-, ninguno de sus órganos subsidiarios (ad hoc) aborda exclusivamente la temática (MERCOSUR, 2019a).

Sin embargo, el panorama no es tan desolador. Aunque quizás atrasado en comparación otras regiones del globo, es innegable los países de América del Sur también vienen buscando una mayor integración a los asuntos ambientales globales, en parte debido a la ya observada vulnerabilidad al cambio climático que tiene la región (CEPAL/ECLAC, 2016). En este sentido, los eventos mencionados anteriormente funcionan como antecedentes para un proceso más extenso de formación y adopción de agenda en torno al cambio climático en la región. En el año 2019, por ejemplo, se realizó el "Primer Encuentro de diálogo político y social sobre Cambio Climático del MERCOSUR" —organizado por su Parlamento Social en el marco de preparación para la COP 25-, además de una Declaración Conjunta de los presidentes del bloque sobre Desarrollo Sostenible (MERCOSUR, 2019b).

En Argentina en particular, se creó recientemente el Gabinete Nacional de Cambio Climático (Decreto 891/2016), el cual busca diseñar políticas públicas coherentes, consensuadas y estratégicas para reducir las emisiones de gases de efecto invernadero y atender el cambio climático. Agrupa ministerios y secretarías nacionales involucrados en políticas climáticas bajo la órbita de Jefatura de Gabinete de ministros. La Secretaría de Cambio Climático y Desarrollo Sustentable es su coordinadora. La participación de las provincias se articula a través del Consejo Federal de Medio Ambiente (COFEMA) y las mesas ampliadas son la instancia de articulación con la sociedad civil, académicos, sector privado y trabajadores.

\footnotetext{
${ }^{7}$ Por ejemplo, cuando uno visita el sitio oficial del bloque, en la sección "Temas" no figura ninguna directamente relacionada al Medioambiente o al cambio climático. Solo existen algunas menciones en torno a lo medioambiental (en general) en otras áreas temáticas como la "Cooperación intrarregional" (Mercosur, 2020) o la salud (Mercosur, 2018).
} 
Un clima para el cambio. Oportunidades y desafíos para la articulación interregional ASEAN-MERCOSUR en materia de cambio climático

DiEgo TOLEDO

Amalia Stuhldreher (2012) menciona diversos cambios producidos en Argentina en materia ambiental en diversos sectores sociales. A nivel público, el INTI y el INTA incorporaron líneas de desarrollo sustentable y energía renovable. También se crearon entidades privadas de investigación; e intereses corporativos como la Sociedad Rural o la Unión Industrial Argentina han creado espacios de "sustentabilidad ambiental". Siguiendo los análisis de María del Pilar Bueno (2017), estos avances se encuadran en una dimensión de "poder climático social" (proceso de institucionalización de ideas, creencias e identidades construidas intersubjetivamente), que junto a otras variables de "poder material climático" (el poder en tanto capacidad material de un Estado y su poder de negociación en un ámbito en particular) explican la capacidad de incidir de un Estado en las negociaciones multilaterales y su doble plexo, interno y externo.

En este marco se desarrolla el proceso de elaboración y validación de los Planes de Acción Sectoriales de Cambio Climático respecto a energía, bosques, transporte, industria, agricultura y ganadería e infraestructura y territorio, que son el piso sobre el cual se desarrolla el Plan Nacional de Mitigación y el Plan Nacional de Adaptación, ambos parte del Plan Nacional de Respuesta al Cambio Climático. En este contexto, los desarrollos tecnológicos y conocimientos científicos que producen las agencias de investigación argentinas pueden realizar importantes aportes a la cooperación sur-sur con los Estados miembros de ASEAN en materia de cambio climático, complementando y fortaleciendo las expectativas abiertas tras la firma del Tratado de Amistad y Cooperación (2017) entre Argentina-ASEAN.

De manera más general, pueden aprovecharse los dispositivos institucionales que existen en los países de varias regiones para potenciar el trabajo en áreas estratégicas para ambas partes. Puede avanzarse en la cooperación sur-sur en cambio climático a través fortalecer una agenda común en los encuentros interregionales, aprovechando espacios como las Reuniones Ministeriales MERCOSUR-ASEAN.

Para ello, podrían establecerse principios de acción (beneficio mutuo, horizontalidad, consulta y consenso) y áreas prioritarias comunes sobre las cuales actuar: educación ambiental (concientización pública), producción mejor adaptada al cambio climático (más limpia), construcción de capacidad de gestión en materia ambiental, cooperación en bienes y servicios ambientales, e intercambio y acuerdo en asuntos globales medioambientales.

Dado el mayor grado de consolidación institucional y de desarrollo de iniciativas que ASEAN tiene en temas de cambio climático, sus aportes podrían ser de gran valor para el bloque sudamericano. Podrían, por ejemplo, pensarse mecanismos de capacitación (foros, reuniones de alto nivel, etc.) para personal encargado de implementar y diseñar políticas ambientales y políticas económicas "más verdes". 
Un clima para el cambio. Oportunidades y desafíos para la articulación interregional ASEAN-MERCOSUR en materia de cambio climático

DiEgo TOLEDO

En relación con la promoción de ciudades sustentables, ambos bloques podrían aprovechar su pertenencia a redes transnacionales (como ICLEI) y la formación de proyectos intrarregionales (como Mercociudades y AIESC) para intercambiar experiencias y fortalecer su capacidad de vincularse entre sí, y nutrir las Iniciativas de ASEAN para el Aire Limpio, Agua Limpia y Tierra Limpia.

En materia de transición energética hacia un modelo bajo en carbón, los desarrollos de Malasia y Tailandia como manufactureros de paneles solares pueden ser objeto de transferencia tecnológica para países como los nuestros, que están comenzando a apostar por las energías renovables. A su vez Brasil, Argentina y Uruguay vienen haciendo fuertes esfuerzos en materia de cooperación energética, apoyándose en espacios como el FOCEM (Fondo de Convergencia Estructural) para el desarrollo de la infraestructura energética (Freier, 2016; Stuhldreher \& Olmos, 2019).

Por último, ambos bloques pueden fortalecer su conocimiento en el campo de la verificación de buenas prácticas agroecológicas y agropecuarias, la promoción de consumos y producciones sustentables, y certificación orgánica y ecológica de productos regionales. MERCOSUR puede aquí realizar un aporte, teniendo en cuenta que desde finales de la década de los 90 busca impulsar la Iniciativa del Sello Verde MERCOSUR (Consani y Servi, 1999; Gillet, 2014). También las Comisiones de Cambio Climático que existen en organismos argentinos como el INTA y el INTI pueden intercambiar conocimiento con agencias homólogas de países de la ASEAN, ejerciendo un rol prominente en desarrollos relativos la seguridad alimentaria interregional y la transformación productiva.

\section{Reflexiones finales}

El panorama político y económico global nos indica que es necesario contar con estrategias de desarrollo que permitan enfocarse en el avance de cada región. No se trata, entonces, de elegir entre Estados Unidos o China. El desafío para ambos bloques es poder mantenerse unidos e integrados frente a la fragmentación de un orden internacional, basado en reglas, que hoy está transitando una crisis. La articulación interregional ASEAN-MERCOSUR es una oportunidad para fortalecer los sistemas de gobernanza global y generar contrapesos alternativos a esos centros de poder hegemónicos, fortaleciendo otros contenidos y canales para la cooperación entre países emergentes. Siendo la ASEAN una de mayores economías del mundo, para el MERCOSUR podría ser un impulso necesario para posicionarse más fuertemente a nivel regional e internacional.

Sin embargo, en el contexto de la actual degradación ambiental global, la reactivación del comercio MERCOSUR-ASEAN no solo puede tratarse de 
Un clima para el cambio. Oportunidades y desafíos para la articulación interregional ASEAN-MERCOSUR en materia de cambio climático

DiEgo TOLEDO

diversificar y agregar valor a las exportaciones de cada región. Las negociaciones comerciales bilaterales e interregionales deben nutrirse del intercambio de conocimiento sobre políticas y formas de mitigar y adaptar las producciones regionales al cambio climático. En la revisión de las iNDCs, los gobiernos de MERCOSUR y ASEAN deben enfatizar el desarrollo tecnológico y medidas efectivas, especialmente en los campos de la energía, el transporte, la seguridad alimentaria y los enclaves urbanos.

La alianza interregional en materia de cambio climático puede ser útil para ejercer una mayor incidencia en las negociaciones multilaterales, especialmente en torno a los mecanismos de financiamiento del cambio climático y los esfuerzos para transformar las matrices productivas. Los países de ambas regiones pueden fortalecer su rol en los regímenes ambientales internacionales alineando sus prioridades nacionales con la agenda global, desarrollando estrategias y modelos de desarrollo económico sustentable más coherentes e integrados.

$\mathrm{Si}$ bien no debemos olvidar que es necesario un esfuerzo mancomunado de actores gubernamentales, privados y sociales para la acción frente al cambio climático, la integración y retroalimentación multinivel de los regímenes regulativos ambientales y su traducción en planes de desarrollo -y de adaptación al cambio climático- nacionales es esencial para generar un clima para el cambio. ASEAN y Mercosur deben recordar que la anarquía ambiental es lo que los Estados hacen de ella.

\section{Referencias bibliográficas}

Association of Southeast Asian Nations (s/f). ASEAN Community Vision 2025. https://www.asean.org/wpcontent/uploads/images/2015/November/aec-page/ASEANCommunity-Vision-2025.pdf

Association of Southeast Asian Nations (2012). ASEAN Action Plan on Joint Response to Climate Change. http://environment.asean.org/wpcontent/uploads/2015/06/ANNEX-8-Lead-Countries-for-ASEANAction-Plan-on-Joint-Response-to-Climate-Change-27-March-2013.pdf Association of SOUTHEAST Asian NATIONS (2015). Fact Sheet - ASEAN Community. https://asean.org/storage/2012/05/7.-Fact-Sheet- onASEAN-Community.pdf

Association Of SOUTHEAST Asian NATIONS (2016). ASEAN Socio-Cultural Community Blueprint 2025. https://asean.org/?static_post=aseansocio-cultural-community-blueprint-2025

Association OF SOUTHEAST Asian NATIONS (2018). ASEAN Multi-sectoral framework for climate change: agriculture and forestry towards food and nutrition seecurity and achievement of SDGs. 
Un clima para el cambio. Oportunidades y desafíos para la articulación interregional ASEAN-MERCOSUR en materia de cambio climático

DIEGO TOLEDO

https://asean.org/storage/2012/05/ASEAN-Multisectoral-

Framework-for-climate-change.pdf

Association Of SOUTHEAST AsIAN NATIONS (2019a). ASEAN Cooperation on Environment: At a glance. https://environment.asean.org/aseancooperation-on-environment-at-a-glance-brochure/

Association of Southeast Asian NATIOns (2019b). Fuelling ASEAN's energy security. Singapore: ISEAS Yusof Ishak Institute.

BALDWIN, R (2011). 21st century regionalism: Filling the gap between 21st century trade and 20th century trade rules. En World Trade Organization (WTO), Economic Research and Statistics Division. No. ERSD -2011 -08. Ginebra

BArtesaghi, I. (2015). La integración regional en Asia Pacífico: El caso de la ASEAN, la APEC y el RCEP. En Phillips Sñanchez, L. (comp), Ágora Latinoamericana. Logros, obstáculos y retos de la integración regional. Tomo I.

BuzAn, B. (2012). Asia: A geopolitical reconfiguration. Politique étrangère, 77(2), pp. 13.

CEPAL/ECLAC (2018). Economics of climate change in Latin America and the Caribean. A graphic view.

CONSANI, N. E., \& SERVI, A. (1999). MERCOSUR y medio ambiente. Relaciones Internacionales.

Del Pilar Bueno, M. (2012). La Anarquía Ambiental es lo que los Estados hacen de Esta. Las Conferencias Ambientales desde Estocolmo a Río+ 20. Latin American Journal of International Affairs, 4(3).

DEL Pilar Bueno, M. (2017). ¿ Poder material o poder social? Reflexiones sobre las negociaciones multilaterales y el poder climático de la Argentina (2003-2015). Relaciones Internacionales, 26(53), 65-86.

FEDOrovsky, J., \& Niglia, G. (2014). La protección del medio ambiente en MERCOSUR y NAFTA. In VII Congreso del IRI/I Congreso del CoFEI/II Congreso de la FLAEI (La Plata, 2014).

FrEIER, A. (2016). Integración energética regional en Mercosur. Los casos de Argentina y Brasil en el área de la energía renovable. En Guzowski, C. (comp.). Políticas de promoción de las Energias Renovables. Experiencias en América del Sur, pp. 153-192. Bahía Blanca: Ediuns.

Fuhr, H., Hickmann, T., \& KERN, K. (2018). The role of cities in multi-level climate governance: local climate policies and the 1.5 C target. Current opinion in environmental sustainability, 30, 1-6.

GILLET, S. (2014). Eco-etiquetado en el MERCOSUR. Potencialidades del Enfoque Regional en la Adopción de Modalidades de Producción y Consumo Sustentables Tesis de Maestría. Facultad de Ciencias Económicas. Universidad de Buenos Aires, Argentina.

HANGGI, H. (2003). Regionalism through interregionalism. East Asia and ASEM. En Fu-Kuo, L. y Philippe, R. (eds.). Regionalism in East Asia: 
Un clima para el cambio. Oportunidades y desafíos para la articulación interregional ASEAN-MERCOSUR en materia de cambio climático

DIEGO TOLEDO

Paradigm shifting? (197-219). Londres: Routledge.

IPCC (2001). Tercer Informe de evaluación. Cambio Climático. pp. 173-199.

HickMAnN, T. (2017). The reconfiguration of authority in global climate governance. International Studies Review, 19(3), pp. 430-451.

Keohane, R. \& Victor, D. (2010). The Regime Complex for Climate Change. Discussion Paper. Cambridge: Harvard Kennedy School.

Kiessling, C. K. (2019). Vinculación entre la política climática doméstica en países emergentes y la política internacional del cambio climático: propuesta de un marco analítico. Comillas Journal of International Relations, (15), pp. 78-98.

Lassa, J. A., \& SEMBIRING, M. (2017). Towards policy integration of disaster risk, climate adaptation, and development in ASEAN: a baseline assessment.

Letchumanan, R. (2010). Climate Change: Is Southeast Asia Up to the Challenge?: is there an ASEAN policy on climate change. En Kitchen, N. (Ed.). IDEAS reports - special reports, 50-62. LSE IDEAS, London School of Economics and Political Science.

Lo Brutto, G., \& GonzÁlez, C. H. (2015). La influencia China en la Cooperación Sur-Sur Latinoamericana, durante la segunda década del Siglo XXI. Cátedra COIBA DT cooperación y desarrollo, 2, pp. 1-15.

MANZANO Franco, K (2016). Mudanças climáticas - o desafio do século (Climate change - the challenge of the century). Konrad Adenauer Foundation.

MERCOSUR (2018) Declaración de los Ministros de Salud del Mercosur y Estados Asociados en relación a la salud y al cambio climático.

MERCOSUR (2019a). Estrategia de gestión del riesgo de desastres de los países del Mercosur.

MERCOSUR (2019b) Declaración Presidencial sobre Desarrollo Sostenible. http://www.puntofocal.gov.ar/doc/decla_eepp_sostenible-12-19.pdf

MERCOSUR (2020) La cooperación intra-MERCOSUR (2005 - 2015): Análisis de siete sectores, pp. 153-155.

ØverLand, I. (2017). Impact of Climate Change on ASEAN International Affairs: Risk and Opportunity Multiplier. Norwegian Institute of International Affairs and Myanmar Institute of International and Strategic Studies. https://www.researchgate.net/publication/320622312_Impact_of_Cl imate_

Change_on_ASEAN_International_Affairs_Risk_and_Opportunity_ Multiplier

PALACIO, G. (2013). Cambio climático, retórica política y crisis ambiental: una nueva interfase entre ciencias naturales y ciencias sociales. En Postigo, J.C. (Ed.) Cambio climático, movimientos sociales y politicas públicas. Una 
Un clima para el cambio. Oportunidades y desafíos para la articulación interregional ASEAN-MERCOSUR en materia de cambio climático

DIEGO TOLEDO

vinculación necesaria (51-74). CLACSO.

PICCONE, T. (2016). The Geopolitics of China's Rise in Latin America. Geoeconomics and global issues (2). Brookings Institution.

RAMON-BerjanO, C. (2019) Globalización con "características chinas". El creciente rol de China en América Latina y el Caribe y sus principales desafíos. Pensamiento Propio, Vol. 49-50, Año 24, pp. 31-52.

Ramoneda, E. (2013). Introducción. En Ramoneda, E. (Ed. Comp.). Concurso de Ensayos ANSEA: Compilación de Ensayos Galardonados 2013-2014. La Plata, Argentina: Instituto de Relaciones Internacionales UNLP.

Rubiolo, M. F. (2011). El Sudeste Asiático y la ASEAN en el escenario económico y político internacional contemporáneo. En Santillán, G.; Mohamed, N.; Morero, N. y Rubiolo, M.F. (Eds.). América Latina y el Este Asiático: Perspectivas desde Córdoba, Argentina: editorial CIECSCONICET-UNC.

Rubiolo, M. F. (2016). Interregionalismo Sureste Asiático-América del Sur: un complemento al bilateralismo. Revista CIDOB d'Afers Internacionals, $N^{\circ} 113$, pp. 211-234.

RÜLAND, J. Y STORZ, C. (2008). Interregionalism and interregional cooperation: the case of Asia- Europe relations. En Rüland, J.; Schucher, G. y Storz, C. (Eds.). Asian-European Relations. Building blocks for global governance (3-31). Nueva York: Routledge.

Ryan, D. (2016). Diseño de la institucionalidad climática: Aportes para el análisis. Ambiente \& Sociedad, 19(4), pp. 249-260.

SalamancA, A. Y NGUYEN, H. (2016). Climate change adaptation readiness in the ASEAN countries. Stockholm Environment Institute - Asia Centre.

Sanahuja, J. A. (2016). Regionalismo e integración en América Latina: de la fractura Atlántico-Pacífico a los retos de una globalización en crisis. Pensamiento propio, 21(44), pp. 29-76.

SANAHUjA, J. A. (2019). La crisis de la integración y el regionalismo en América Latina: giro liberal-conservador y contestación normativa. Anuario Ceipaz. 2018-2019. Ascenso del nacionalismo y el autoritarismo en el sistema internacional, pp. 107-126.

Stockholm EnVIRONMENT InstituTE - Asia (2016). Climate change adaptation readiness in the ASEAN countries. Chulalongkorn University.

STUHLDREHER, A. (2012). Construcción participativa del regionalismo estratégico: ¿hacia una agenda medioambiental externa del Mercosur? Revista Brasileira de Política Internacional, 55(1), 194-210.

Stuhldreher, A., \& Olmos, V. M. (2019). Desarrollo sostenible, cambio climático y comercio de energía en el contexto regional del MERCOSUR: consideraciones desde Uruguay. Latin American Journal 
Un clima para el cambio. Oportunidades y desafíos para la articulación interregional ASEAN-MERCOSUR en materia de cambio climático

\section{of Trade Policy, (5), 65.}

TussiE, D. (2019). La reconfiguración global: Su capítulo en vivo para América Latina. En Altmann Borbón, J. (Ed.), América Latina frente a la reconfiguración global (pp. 105-123). Buenos Aires: FLACSO.

ViglizzO, E. F. \& RiCARD, M.F (S/F). Emisiones, secuestro y balance de carbono en ambientes rurales del Mercosur: en busca del "eslabón perdido" de carbono.

Viglizzo, E. F. (2018). Cambio climático y seguridad alimentaria global: Oportunidades y amenazas para el sector rural argentino. Anales de la ANAV, 69.

ZELICOVICH, J. (2016). El MERCOSUR frente al "Regionalismo del siglo XXI". Algunas claves para la comprensión del devenir del proceso de integración. Revista Aportes Para La Integración Latinoamericana, (34), pp. 1-27. https://revistas.unlp.edu.ar/aportes/article/view/3539 
Comité de ASEAN en Buenos Aires

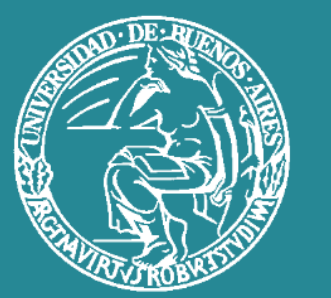

Grupo de Estudios sobre Asia y América Latina Instituto de Estudios de América Latina y el Caribe Universidad de Buenos Aires 